\title{
The antihyperlipidemic effects of fullerenol nanoparticles via adjusting the gut microbiota in vivo
}

\author{
Juan Li ${ }^{1}$, Runhong Lei ${ }^{1}$, Xin Li ${ }^{2}$, Fengxia Xiong ${ }^{1}$, Quanyang Zhang ${ }^{2}$, Yue Zhou ${ }^{2}$, Shengmei Yang ${ }^{1}$, Yanan Chang ${ }^{1}$, \\ Kui Chen ${ }^{1}$, Weihong $\mathrm{Gu}^{1}$, Chongming $\mathrm{Wu}^{2^{*}}$ and Gengmei Xing ${ }^{1 *}$ (i)
}

\begin{abstract}
Background: Nanoparticles (NPs) administered orally will meet the gut microbiota, but their impacts on microbiota homeostasis and the consequent physiological relevance remain largely unknown. Here, we describe the modulatory effects and the consequent pharmacological outputs of two orally administered fullerenols NPs (Fol1 $\mathrm{C}_{60}(\mathrm{OH})_{7}(\mathrm{O})_{8}$ and Fol113 $\left.\mathrm{C}_{60}(\mathrm{OH})_{11}(\mathrm{O})_{6}\right)$ on gut microbiota.

Results: Administration of Fol1 and Fol113 NPs for 4 weeks largely shifted the overall structure of gut microbiota in mice. The bacteria belonging to putative short-chain fatty acids (SCFAs)-producing genera were markedly increased by both NPs, especially Fol1. Dynamic analysis showed that major SCFAs-producers and key butyrate-producing gene were significantly enriched after treatment for 7-28 days. The fecal contents of SCFAs were consequently increased, which was accompanied by significant decreases of triglycerides and total cholesterol levels in the blood and liver, with Fol1 superior to Fol113. Under cultivation in vitro, fullerenols NPs can be degraded by gut flora and exhibited a similar capacity of inulin to promote SCFA-producing genera. The differential effects of Fol1 and Fol113 NPs on the microbiome may be attributable to their subtly varied surface structures.
\end{abstract}

Conclusions: The two fullerenol NPs remarkably modulate the gut microbiota and selectively enrich SCFAproducing bacteria, which may be an important reason for their anti-hyperlipidemic effect in mice.

Keywords: Fullerenols, Gut microbiota, Short-chain fatty acid (SCFA), Blood lipids, Butyrate-producing bacteria

\section{Background}

Nowadays, an increasing body of nanomaterials are developed as new therapeutic and diagnostic tools for many serious diseases [1]. The prominent therapeutic effects of nanodrugs on cancers [2], angiogenesis inhibition [3] antiinflammation [4] and other conditions have been demonstrated through animal, cellular, and biomolecular experiments $[5,6]$. Oral administration is a preferred route for clinical compliance of nanodrugs which increases the likelihood that nanomaterials encounter microbes in the gastrointestinal (GI) tract.

\footnotetext{
* Correspondence: cmwu@implad.ac.cn; xinggm@ihep.ac.cn

${ }^{2}$ Pharmacology and Toxicology Research Center, Institute of Medicinal Plant Development, Chinese Academy of Medical Sciences \& Peking Union Medical College, Beijing 100193, China

${ }^{1}$ CAS Key Laboratory for Biomedical Effects of Nanomaterial \& Nanosafety, Institute of High Energy Physics, Chinese Academy of Science (CAS), Beijing 100049, China
}

Therefore, what occurs when nanomaterials meet gut microbes is an important issue worthy of exploring.

There are more than 100 trillion microbes living in our digestive tracts which are collectively called the gut microbiota. A growing body of evidence indicates that the gut microbiota is indispensable to the maintenance of human health [7]. Structural changes in the gut microbiota are closely associated with various diseases, ecially metabolic disorders such as obesity, insulinistant diabetes, and nonalcoholic fatty liver disease $[8$, investigations revealed that the therapeut ine [11], and Ganoderma lucidum [12] are at least partially attributable to their modulation of gut microbiota, demonstrating the powerful capacity of the gut microbiome to mediate the safety and efficacy of traditional oral drugs $[13,14]$. The impacts of nanoparticles 
(NPs) on the gut microbiota have also attracted increasing attention. The nanoparticles of titanium dixoide $\left(\mathrm{TiO}_{2}\right)$, Zinc and silver have been reported to be able to change the gut microbial community, but their modulating effects on the gut microbiota remain controversial among different research groups [15-20].

Fullerenols are polyhydroxylated C60 with excellent properties such as easy to be obtained, high water solubility and good biocompatibility [4, 21, 22]. Fullerenol NPs are now widely used in biological and medical researches due to their various biological activities. Previous studies have shown that fullerenol NPs can inhibit the growth of various microbes in vitro [22, 23], but their in vivo impact on gut microbes is largely unknown. Therefore, fullerenol NPs are a suitable condidate to investigate the modulating role of nanomaterials on the gut microbiota and the consequent physiological relevance.

In the present study, we investigated the impacts of two fullerenol NPs $\left(\right.$ Fol1 $\mathrm{C}_{60}(\mathrm{OH})_{7}(\mathrm{O})_{8}$ and Fol113 $\left.\mathrm{C}_{60}(\mathrm{OH})_{11}(\mathrm{O})_{6}\right)$ on gut microbiota homeostasis in vivo. We also assessed the influences of fullerenol NPs on the SCFA-producing bacteria levels, fecal SFCA concentration, and lipid levels in blood and liver to establish a physiological association between the gut microbe-regulating roles of fullerenols and their pharmacological benefits. Lastly, we examined the impacts of fullerenol NPs on SCFA-producers in vitro and compared them with those of inulin to clarify how fullerenols NPs modulate the gut microbiome and exert antihyperlipidemic effects.

\section{Methods}

\section{Preparation of fullerenols}

Fullerenols (Fol 1) were synthesized by the alkaline reaction [24]. Briefly, a solution of $\mathrm{C}_{60}$ in toluene was added to an aqueous solution containing $\mathrm{NaOH}$ and $40 \%$ tetrabutylammonium hydroxide (TBAH) as catalyst. The mixture was stirred at room temperature for $24 \mathrm{~h}$. The color of the solution changed from deep violet to colorless, while a brown sludge precipitated on the bottom of the beaker. The aqueous phase was then separated and evaporated under vacuum to obtain the crude product, which was further washed by methanol. The crude product was then passed through a Sephadex G-25 column and eluted with double distilled water. The difference of the preparations of Fol 113 was used $\mathrm{H}_{2} \mathrm{O}_{2}$ $30 \%$ was as initiators in the stirring mixture.

Purified fullerenols were prepared by a previously reported method in our laboratory [24]. Dry samples were analyzed on a Nicolet Magna-IR750 FTIR spectrophotometer equipped with a Nic-plan IR microscope. Scanning electron microscope (SEM) imaging was performed using a HITACH 4800 s (Japan) instrument. Dynamic light scattering(DLS) was monitored the hydrodynamic sizes and zeta potentials of fullerenol nanoparticles in saline, simulated gastric and intestinal solusion by Malvern Zetasizer Nano ZS instrument at the concertration of $500 \mu \mathrm{g} / \mathrm{mL}$ and the solusions were prepared according to National Formulary [25]. The samples were deposited onto silica substrates for SEM and onto high-purity gold substrates for XPS. Electronic properties of the samples were studied by XPS at the photoelectron station of Beijing Synchrotron Radiation Facility of the Chinese Academy of Sciences. The XPS measurements in an ultra-vacuum chamber with background pressure ranging $8 \times 10^{-10}$ to $1 \times 10^{-9}$ Torr and photon energy of $400.0 \mathrm{eV}$. The assay resolution was estimated to be $\sim 0.5 \mathrm{eV}$. All electron spin resonance (ESR) experiments were carried out by using a JES-FA200 $\mathrm{X}$-band ESR spectrometer (JEOL, Japan), at Tsinghua University in China.

\section{Modulation of gut microbiota by fullerenols in mice}

All the animal experiments were performed in accordance with the National Institutes of Health regulations for the care and use of animals in research and were approved by the Medical Ethics Committee of Peking Union Medical College (No. YZS201603004).Twentyfour male specific pathogen-free (SPF) C57BL/6 mice (8week-old, 22-26 g) were obtained from Vital River Laboratory Animal Technology Co., Ltd. (Beijing, China). Animals were kept in a humidity-controlled room on a 12-h light-dark cycle with food and water available ad libitum for 1 week. The mice were then divided randomly into 3 groups (8 animals per group) and fed with normal diet. The Fol1 and Fol113 groups were gavaged with Fol1 or Fol113 NPs $(20 \mathrm{mg} / \mathrm{kg}$ per day, suspended in distilled water) for a month, while the control group (NC) was given an equal volume of distilled water. Fresh feces were collected from each mouse at $2 \mathrm{~h}$ after the last gavage, snap-frozen in liquid nitrogen then stored at $-80{ }^{\circ} \mathrm{C}$ for subsequential analysis.

\section{Fullerenol NPs distribution and excretion in mice}

The In vivo distribution of fullerenol NPs were measured by PET imaging using ${ }^{64} \mathrm{Cu}$-labeled fullerenol NPs $\left({ }^{64} \mathrm{Cu}-\mathrm{C}_{60}\right)$ as described in previous study [26]. The ${ }^{64} \mathrm{Cu}-\mathrm{C}_{60}$ nanoparticles (25.9 MBq) dispersed in $100 \mu \mathrm{L}$ saline were given to the mice by single oral administration $(n=3)$. The mice were anesthetized with $1.5 \%$ isoflurane for imaging at $0.5,1,2$ and $6 \mathrm{~h}$. The data were acquired using a small animal PET scanner (Eplus-166, Institute of High Energy Physics, Chinese Academy of Sciences). The PET images were corrected for detector efficiency, dead-time, decay, photon scatter, and attenuation. The control groups were injected with $25.9 \mathrm{MBq}$ ${ }^{64} \mathrm{CuCl}_{2}$ and subjected to the same PET protocol. 
To assess the excretion of fullerenol NPs, another animal experiment was performed as described above. Animals were given ${ }^{64} \mathrm{Cu}-\mathrm{C}_{60}$ nanoparticles (25.9 MBq) dispersed in $100 \mu \mathrm{L}$ saline by a single oral administration $(n=3)$. These animals were kept in metabolism cages for $24 \mathrm{~h}$ after material gavage. Feces and urine samples were collected at 2, 4, 6 and $24 \mathrm{~h}$ and counted in a CAPRAC-t well-type counter (Capintec, Inc., Pittsburgh, PA, USA).

\section{In vitro single-batch fermentation}

Fresh fecal materials were obtained from healthy C57 mice, pooled together, and suspended in $0.1 \mathrm{M}$ sterile PBS (pH 7.0) to prepare a $10 \% w / v$ fecal slurry. An aliquots of $5 \mathrm{~mL}$ fecal slurry was inoculated into each $100 \mathrm{~mL}$ fermentation vessel that contained $15 \mathrm{~mL}$ of the modified Broth medium (Hopebio Ltd., China). The Fol 1 and Fol 113 NPs were added into the vessels with a final concentration of $0.1 \mathrm{mg} / \mathrm{mL}$. As positive control, inulin, a well-known prebiotics that is able to promote short-chain fatty acid (SCFA)-producing bacteria [27], was added into the vessels to achieve a final concentration of $5 \mathrm{~g} / \mathrm{L}$. The cultures were fermented for $48 \mathrm{~h}$ in the micro-anaerobic incubation system (INVIVO 400, Ruskin Technologies, UK). After culture, the bacteria were collected by 12,000 rpm centrifugation for $20 \mathrm{~min}$. The cell pellets were used for MALDI-TOF-MS and real-time quantitative PCR analysis. The experiment were performed in triplet.

\section{Phylogenetic analysis}

Fecal DNA samples of each mouse extracted using a FastDNA $^{\oplus}$ Spin Kit for Stool (MP Biomedicals, Santa Ana, USA) was then amplified by barcoded composition primers flanking the V3/V4 regions of the $16 \mathrm{~S}$ rRNA gene. The composite primers consist of BCP-F (50GCCTTGCCAGCCCGCTCACACTCCTACGGRAGGC AGCAG-30; the underlined sequence indicates the target region) and BCP-R (50-GCCTCCCTCGCGCCAT CAG-X-TACNVGGGTATCTAATCC-30; "X" denotes the unique barcode for each sample). The PCR reaction was performed as follows: $95^{\circ} \mathrm{C}$ for $5 \mathrm{~min}$, followed by 30 cycles of $95{ }^{\circ} \mathrm{C}$ for $30 \mathrm{~s}, 55^{\circ} \mathrm{C}$ for $30 \mathrm{~s}$ and $72{ }^{\circ} \mathrm{C}$ for $30 \mathrm{~s}$, with a final extension at $72{ }^{\circ} \mathrm{C}$ for $5 \mathrm{~min}$. The amplicons were pooled and purified using a QiaQuick PCR purification kit (Qiagen, Valencia, USA). The mixed PCR products were sequenced on a 454 FLX pyrosequencer platform (Roche, Branford, CT) according to manufacturer's instruction. Pyrosequencing data analysis was carried as previously described [28].

\section{SCFA analysis}

Fecal SCFAs were evaluated by a gas chromatography mass spectrometer (GC-MS). Briefly, $200 \mathrm{mg}$ of frozen feces was suspended in $1 \mathrm{~mL}$ of $1 \% \mathrm{HCl}$ and strongly vortexed for $1 \mathrm{~min}$. 2-Ethylbutyric acid (Sigma Aldrich, USA) was added as internal standard in a final concentration of $2 \mathrm{mM}$. The samples were centrifuged at $5000 \times \mathrm{g}$ for $5 \mathrm{~min}$ and the supernatant was acidified to $\mathrm{pH} 0$ with $\mathrm{HCl}(10 \mathrm{~mol} / \mathrm{L})$. Each sample was extracted at $4{ }^{\circ} \mathrm{C}$ using an equal volume of diethyl ether. Aliquots $(80 \mu \mathrm{L})$ of extracts were added with $16 \mu \mathrm{L} \mathrm{N}$-tertbutyldimethylsilyl-N methyltrifluoroacetamide (Sigma Aldrich) and incubated at $40{ }^{\circ} \mathrm{C}$ for $2 \mathrm{~h}$. The SCFAs contents of each samples were analyzed on an Agilent 5975C GC-MS (Agilent Technologies, Palo Alto, CA, USA) equipped with a HP-5MS column $(0.25 \mathrm{~mm} \times$ $30 \mathrm{~m} \times 0.25 \mu \mathrm{m})$ and a 5973 Network Mass Selective Detector. The GC program was performed as follows: started at $40{ }^{\circ} \mathrm{C}$, heated to $70{ }^{\circ} \mathrm{C}$ by $5{ }^{\circ} \mathrm{C} / \mathrm{min}$ and held for $3.5 \mathrm{~min}$, then ramped at $20{ }^{\circ} \mathrm{C} / \mathrm{min}$ to $160{ }^{\circ} \mathrm{C}$ followed by $35{ }^{\circ} \mathrm{C} / \mathrm{min}$ to $280{ }^{\circ} \mathrm{C}$ and held for $3 \mathrm{~min}$. The $\mathrm{m} / \mathrm{z}$ ratios of monitored ions were as follows: 117 (acetate), 131(propionate), 145 (butyrate), and 173 (2-ethylbutyric acid). SCFAs were quantified with a five-point calibration curve.

\section{Dynamic modulation of microbiota and lipids in body by fullerenols}

C57BL/6 male mice (8-week-old) were kept in a humidity-controlled room on a 12-h light-dark cycle with food and water available ad libitum. The mice were then divided randomly into 3 groups (8 animals per group) and fed with high-fat diet (HFD) which contained $60 \%$ fat, $14 \%$ protein, and $26 \%$ carbohydrate and provided a total energy content of $21.0 \mathrm{~kJ} / \mathrm{g}$. Fol1 and Fol113 NPs $(20 \mathrm{mg} / \mathrm{kg}$ per day, suspended in distilled water) was administrated via oral gavage for 3 weeks. The control group was given equal volumn of distilled water only. Fecal samples were taken from each mouse on $0,3,7,14$ and 28 day after fullerenols treatment. Total bacteria number and the relative abundance of specific bacteria (Allobaculum spp., Clostridium cluster $I V$ and $X I V a$ ) as well as the relative abundance of butyrate-producing genes (BcoA and Buk) in the fecal were determined as described below. After the last fecal samples were acquired, mice were anesthetized in chambers saturated with isoflurane and sacrificed by cardiac puncture. Blood and liver tissue were taken for the measure of serum and liver levels of triglycerides (TG) and total cholesterol (TC) by respective kits (BioSino Co., Ltd., Beijing, China).

\section{Realtime PCR}

Real-time quantitative polymerase chain reaction (realtime PCR) was performed on a 7500 Real-Time PCR System (Applied Biosystem) to quantify the abundance of specific bacteria and butyrate-producing genes. The 
gene-specific primers were designed to target the total, specific bacteria (Allobaculum spp., Bifidobacterium spp., Clostridium clusters IV and XIVa), butyrateproducing genes (butyryl coenzyme A transferase (BcoA) and butyrate kinase (Buk)) (Additional file 1: Table S2). Cloned 16S rRNA genes of $E$. coli were used to construct standard curve for total bacteria copies. The total bacteria were expressed as $\log 10$ copies (16 S DNA gene)/g feces. The relative abundance of specific bacteria or functional genes was normalized to the total bacteria.

\section{Histologic analysis}

A piece of liver was taken from each animal and fixed in $4 \%$ buffered neutral formalin for at least 2 days. Then liver samples were embedded in paraffin and cut at $4 \mu \mathrm{m}$. The sections were stained with hematoxylin and eosin (H\&E), and their morphological changes were evaluated. The steatotic level of liver was assessed by the fatty degeneration (ballooning) of hepatocytes. At least 15 slides of each group were analyzed.

\section{Quantitative analysis of fullerenols by MALDI-TOF-MS}

The polyhydroxyl $C_{70}$ were as reference to quantify the fullerenols of $\mathrm{C}_{60}$ by MALDI-TOF-MS without matrix (Matrix-Assisted Laser Desorption/Ionization Time of Flight Mass Spectrometry) (UltrafleXtreme, Bruker, Germany). The standard curve of $\mathrm{C}_{60} / \mathrm{C}_{70}$ and the concentration of $C_{60}$ were obtained and the bacteria suspension samples were analysed under the same condition.

\section{Statistics}

Data are presented as the means \pm SEM. SPSS 17.0 software was used for the statistical analysis. To analyze the correlation between the abundance of different gut microbe and SCFAs level, multiple-testing corrected pairwise Spearman correlation analysis was performed on the relative abundance of individual genus and the respective fecal SCFA content in each animal, as described previously [29]. The significance of group differences for normally distributed data was assessed by one-way ANOVA followed by Tukey post hoc tests. $P<0.05$ was considered statistically significant.

\section{Results and discussion}

\section{Physicochemical characterization of fullerenol NPs}

Fol1 and Fol113 NPs were synthesized by different routes and purified using an adapted method previously established in our laboratory [24]. Both NPs are stable in aqueous solution for several months (Additional file 1: Figure S1a). Scanning electron microscopy (SEM) images showed that Fol1 and Fol113 have aggregation states, with $\mathrm{d}=100 \pm 13 \mathrm{~nm}$ and $90 \pm 5 \mathrm{~nm}$, respectively (Fig. 1a). The hydrodynamic sizes of Fol113 NPs were also smaller than Fol1 as determined by dynamic light

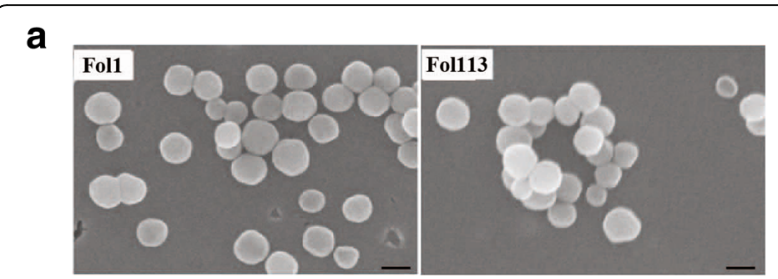

b
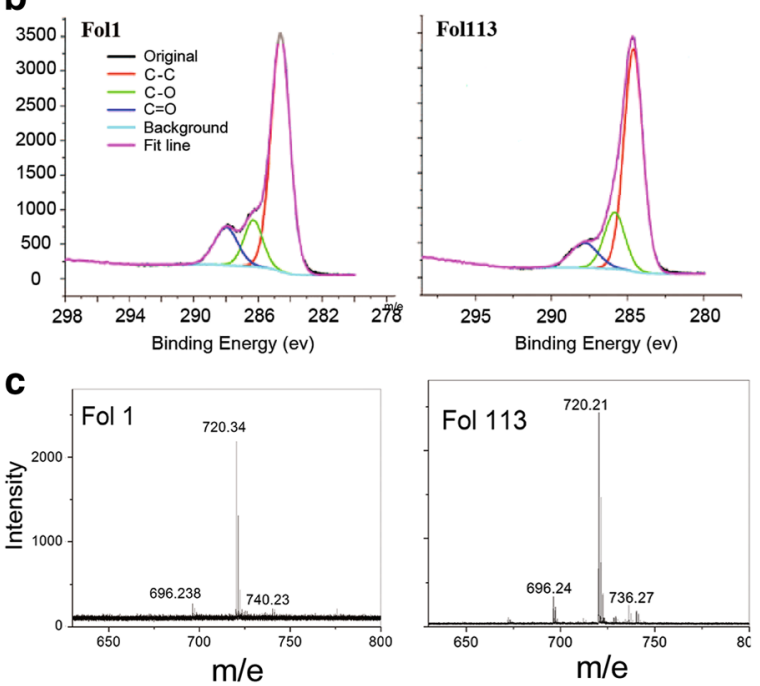

Fig. 1 The physiochemical characterizations of fullerenols. a SEM images of NP aggregates ( $b a r=100 \mathrm{~nm})$, b XPS data, c MALDI-TOFMS data without a matrix

scattering experiments (Additional file1: Table S1) in saline, simulated gastric and intestinal solutions. The zeta potentials and hydrodynamic sizes of Foll and Fol113 indicated good stability in the GI tract. The ultraviolet and Fourier-transform infrared spectroscopy (FTIR) data in Additional file1: Figure S1 indicated that the fullerene cages have similar groups and structures. However, the X-ray photoelectron spectroscopy (XPS) data (Fig. 1b) indicated different types of functionalized carbon atoms on the carbon cages. With a general formula of $\mathrm{C}_{60}(\mathrm{OH}) \mathrm{m}(\mathrm{O}) \mathrm{n}$, Fol1 should be $\mathrm{C}_{60}(\mathrm{OH})_{7}(\mathrm{O})_{8}$ and Fol113 should be $\mathrm{C}_{60}(\mathrm{OH})_{11}(\mathrm{O})_{6}$ according to the XPS data (Table 1). The characteristic fullerene peak of $720(\mathrm{~m} / \mathrm{e})$ was detected in both fullerenol NPs on MALDI-TOF-MS which kept the features of fullerenes (Fig. 1c).

Table 1 Comparison of relative peak areas in the XPS spectra of fullerenols

\begin{tabular}{lcccl}
\hline NPs & C-C (\%) & C-O (\%) & $\mathrm{C}=\mathrm{O}(\%)$ & $\begin{array}{l}\text { Formula }(\mathrm{C60}(\mathrm{OH}) \mathrm{m}(\mathrm{O}) \mathrm{n}) \\
(\mathrm{calculated})\end{array}$ \\
\hline Fol 1 & 71.66 & 13.97 & 14.37 & $\mathrm{C60}(\mathrm{OH}) 7(\mathrm{O}) 8$ \\
Fol 113 & 69.31 & 19.30 & 11.39 & $\mathrm{C60}(\mathrm{OH}) 11(\mathrm{O}) 6$ \\
\hline
\end{tabular}


Fullerenol NPs markedly change the gut microbiota structure To assess the impacts of fullerenol NPs on gut microbiota, C57BL/6 mice were treated with Fol1 or Fol113 NPs $(20 \mathrm{mg} / \mathrm{kg} /$ day, oral administration) for 28 days, and distilled water-treated mice were used as a negative control (NC). Fresh feces samples were collected from each mouse within $2 \mathrm{~h}$ after the last gavage and sent for $16 \mathrm{~S}$ rRNA pyrosequencing. The bar-coded pyrosequencing provided 1,150,372 usable pyrosequencing reads $(189,302$ operational taxonomic units [OTUs]) from 24 fecal samples. After discarding the singleton OTUs that could have been due to sequencing error, 20,750 nonsingleton OTUs $(3537 \pm 855$ OTUs per sample) were recovered for phylogenetic analysis. Rarefaction and Shannon-Wiener curves showed that although new, rare phylotypes would be expected with additional sequencing, most gut microbial diversity was captured (Additional file 1: Figure S2a and S2b).
Oral administration of Fol1 or Fol113 NPs for 28 days remarkably shifted the overall structure of gut microbacteria in vivo. Firstly, principal component analysis (PCA) (Fig. 2a), principal coordinate analysis (PcoA) (Fig. 2b), and UniFrac distance-based hierarchical clustering analysis (Fig. 2c) were used to monitor overall structural changes in the gut microbiota. The results show that both fullerenol NPs markedly shifted structure. Most samples of Fol1 and Fol113 groups were completely separate from the control group along Principal component/coordinate 1 (PC1/PCoA1) $(P<0.05)$ except for a few overlaps (Fig. 2a and b). Hierarchical clustering analysis also revealed remarkable modulatory effects of the fullerenol NPs on gut microbiota structure. The gut flora modulated by Fol1 and Fol113 were largely separate from the NC group, but there were some overlaps (Fig. 2c).

Venn diagram analysis confirmed marked separation of the gut microbes by Fol1 and Fol113 NPs at the OTU

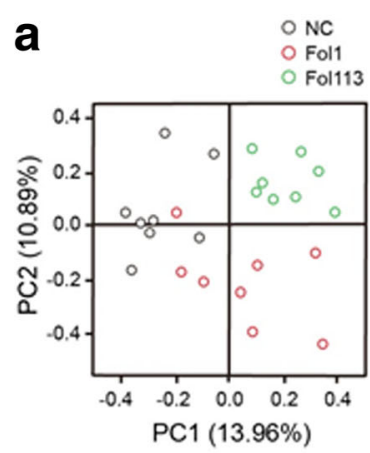

C

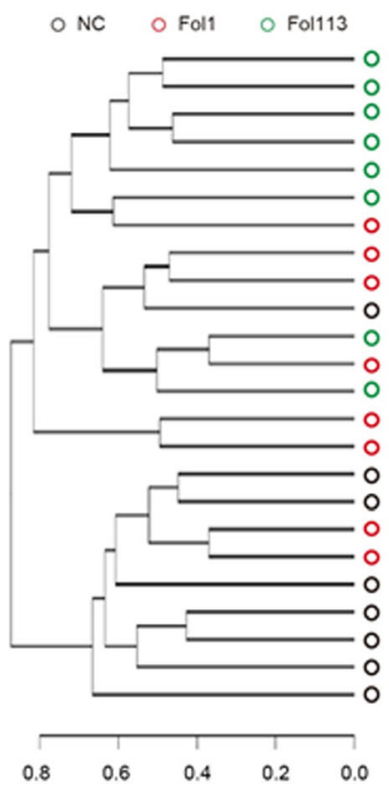

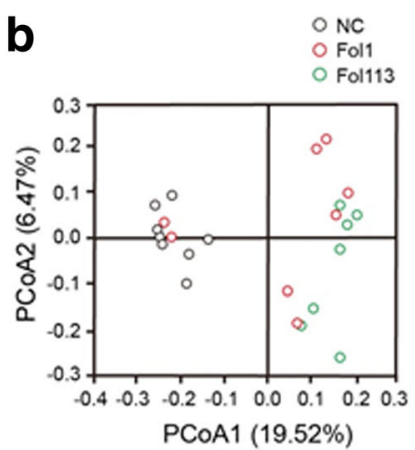

d

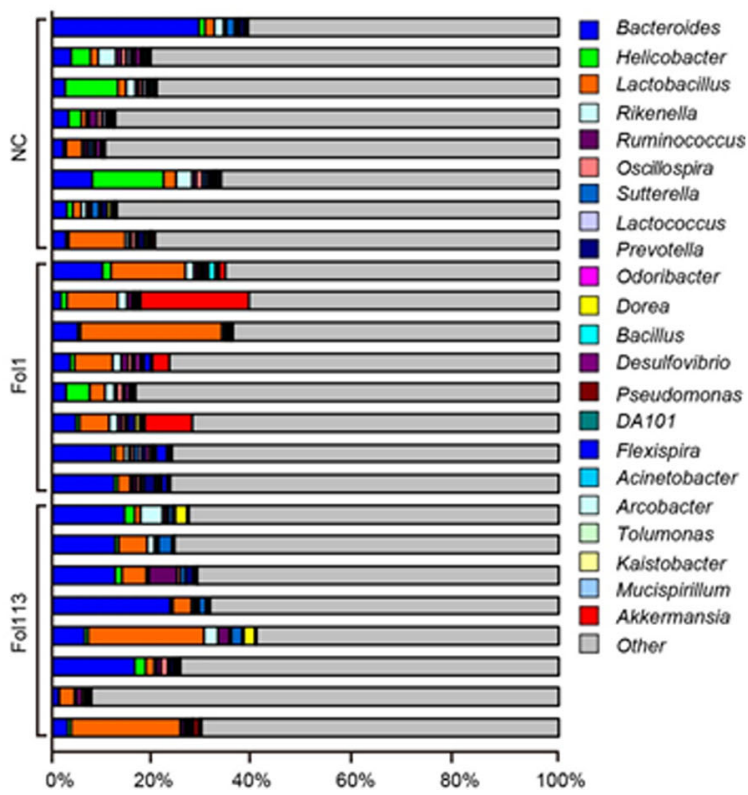

Fig. 2 Responses of the gut microbiota structures to fullerenol NPs. a PCA score plot, $\mathbf{b}$ PCoA score plot, $\mathbf{c}$ clustering of the group means based on the Mahalanobis distances calculated using MANOVA about unweighted UniFrac PcoA, d proportion of the 22 most abundant genera in each animal 
level. As shown in Additional file 1: Figure S3, there were a total of 4328 OTUs in all three groups. Those treated with Fol1 or Fol113 NPs shared 8707 (4379+ $4328)$ and $6547(2219+4328)$ OTUs with the NC group, respectively. However, there were 6423 OTUs exclusive to NP-treated animals (Additional file 1: Figure S3). Additional file 1: Figure S4 displays the relative abundance of the top 50 OTUs in each animal, and NPs enriched those belonging to the genera Lactobacillus, Akkmansia, and Allobaculum. As the three genera are known to be beneficial for lipid metabolism, this suggested that Fol1 and Fol113 NPs could be useful for treating metabolic diseases such as obesity and hyperlipidemia.

Taxon-based analysis also revealed large changes in gut microbial composition induced by Fol1 and Fol113 NPs. A total of 17 in 42 phyla, 52 in 122 classes, 86 in 236 orders, 173 in 420 families, and 252 in 727 genera were significantly different among the NC and NPtreated groups $(P<0.05$, Additional file 2: Table S3). Although both fullerenol NPs remarkably changed the relative abundance of numerous types of bacteria, the significantly changed phylotypes accounted for only a minor part of the total gut microbiota. The five phyla (Acidobacteria, Gemmatimonadetes, Nitrospirae, TM6, and Thermi) significantly changed by both fullerenols NPs represented $0.602 \%$ of the total gut bacteria in the NC group. For major phyla in the gut microbiome, Fol1 and Fol113 NPs exerted minimal influence on the abundance of Firmicutes and Bacteroidetes, but both moderately decreased the relative abundance of Proteobacteria that is known to contain multiple opportunistic pathogens (Additional file 2: Table S3). At the genus level, the two fullerenols showed enriching effects on Lactobacillus, Dorea, Bifidobacterium, Allobaculum, Blautia, Parabacteroides, Akkermansia, and Anaerotruncus (Fig. 2d and Additional file 2: Table S3), all of which are known SCFA producers. ${ }^{26}$ Interestingly, although none of the eight SCFA-producing genera was significantly enriched by either fullerenol NP, their total abundance in each animal was significantly increased by Fol1 NPs $(17.30 \%$ vs $3.45 \%, P<0.05)$ and almost significantly by Fol113 NPs $(10.63 \%$ vs $3.45 \%, P=0.077)$. Although further investigations, especially the dose-dependent effects of fullerenol NPs on the gut microbiota, are still needed, these results provided clear evidence that fullerenol NPs can modulate gut microbiota.

Dynamic analysis showed that in addition to changing the static state of gut microbiota, Fol1 and Fol113 NPs modulated microbial composition dynamically. The dynamic experimental groups were treated with Fol1, Fol113, or distilled water, and fecal samples from each mouse were collected on day $0,3,7,14$, and 28 . Total bacteria number, the relative abundance of SCFA- producing bacteria (Allobaculum spp., Clostridium cluster $I V$, and XIVa), and the relative abundance of butyrate-producing gene (butyryl coenzyme A transferase $[B c o A])$ were determined by real-time quantitative polymerase chain reaction. Compared to $\mathrm{NC}$, oral administration of Fol1 and Fol113 NPs did not remarkably decrease the total numbers of gut bacteria but increased SCFA-producing bacteria such as Clostridium clusters $I V$ and Allobaculum spp., as well as the relative abundance of $\mathrm{BcoA}$ after treatment for 7 days (Fig. 3a-e). These results were in accordance with $16 \mathrm{~S}$ rRNA sequencing and indicate that the modulatory effects of fullerenol NPs on SCFA producers and genes are time dependent.

\section{Fullerenol NPs enhance fecal SCFA content and decrease blood and liver lipids}

SCFAs are predominantly byproducts of the fermentation of non-digestible dietary fibers through the action of intestinal anaerobic bacteria (see Fig. 4a). Acetate, propionate, and butyrate represent the most abundant $(\geq 95 \%)$ metabolite molecular species of intestinal SCFAs [30]. As the total abundance of main SCFA-producing genera was markedly enriched by both NPs, especially Fol1, we determined fecal acetate, propionate, and butyrate levels by gas chromatography-mass spectrometry.

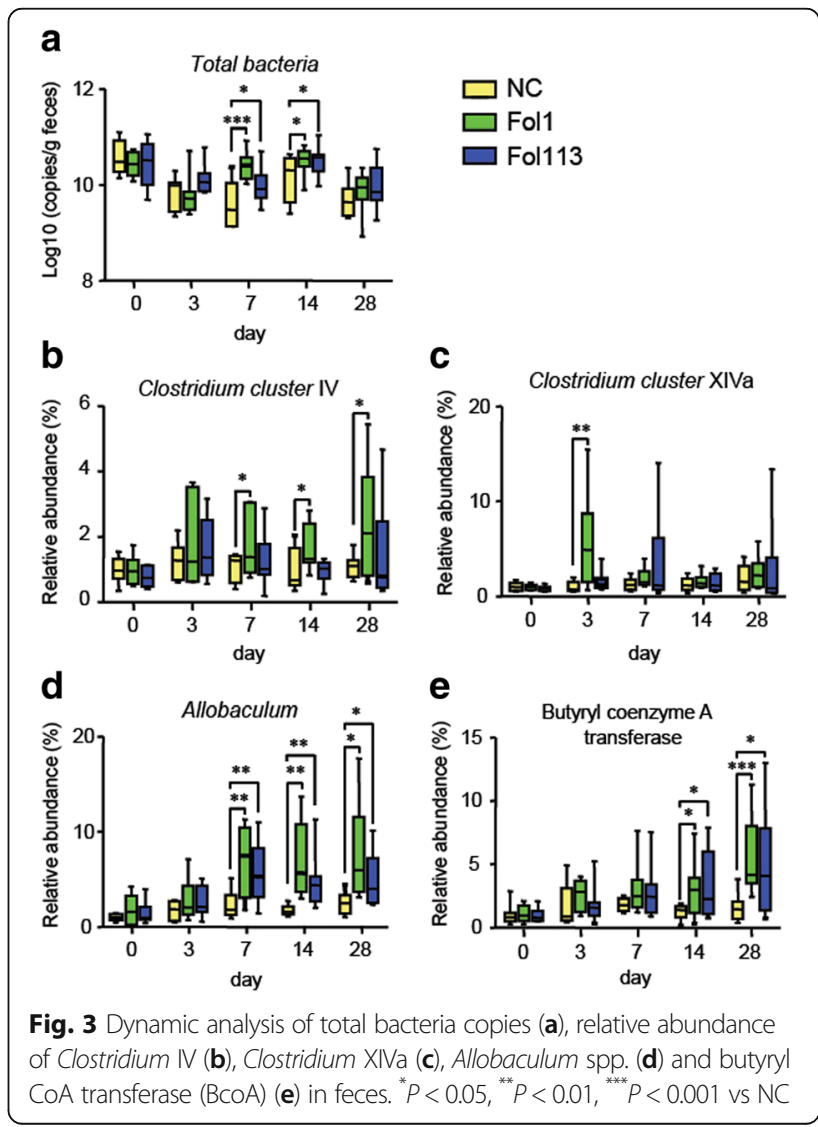



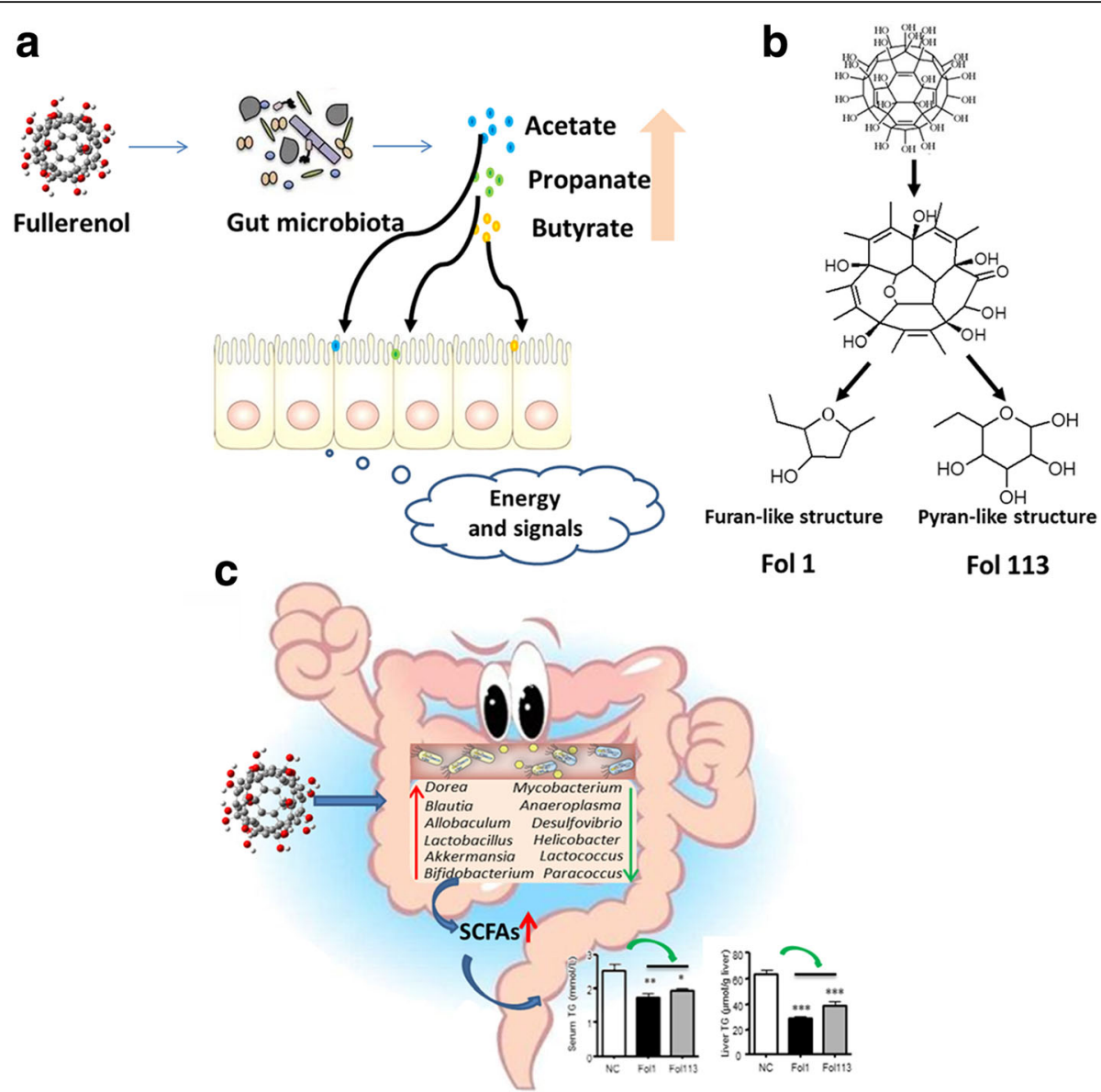

Fol 113

Fig. 4 a Fullerenol NPs ameliorate hyperlipidemia by modulating gut microbiota structure and increasing SCFA production. b Fullerenol NPs contain furan- and pyran-like structures that could be used by gut microbes

The fecal contents of all three were significantly increased by Fol1 NPs as compared with the NC group (Fig. 5a-c). Fol113 NPs exerted a similar enriching effect on the three major SCFAs, but the increases were not statistically significant except for acetate (Fig. 5a-c).

We further performed correlation analysis on the relative abundance of individual genera in each animal with their respective fecal SCFAs contents. The abundance of genera Lactobacillus, Allobaculum, Bifidobacterium, Dorea, and Blautia strongly positively correlated with fecal SCFAs levels (Fig. 5d), which was in accordance with their known SCFA-producing functions. Treatment with fullerenol NPs enriched all five genera (Fig. 5e), suggesting that the increases of Lactobacillus, Allobaculum, Bifidobacterium, Dorea, and Blautia might be linked to the SCFA-promoting effect of fullerenol NPs. In addition to promoting SCFA-producing microbe proliferation, the fecal SCFA concentration was also significantly increased by Fol1, and to a lesser extent by Fol113.

SCFAs, especially butyrate, are key regulators of host energy homeostasis and help prevent adiposity and hyperlipidemia. To confirm the correlation between gut microbiota modulation by fullerenol NPs and pharmacological outcomes, we evaluated the effects of Fol1/ Fol113 NPs on blood and liver lipid levels. In accordance with their SCFA-promoting function, treatment with Fol1 or Fol113 NPs significantly decreased serum and liver levels of total cholesterol (TC) and triglycerides (TG), with Fol1 being more effective (Fig. 6a-d). Accordingly, the high-fat diet (HFD)-induced fatty degeneration (ballooning) of hepatocytes was largely attenuated by both NPs (Fig. 6e), suggesting that Fol1/ Fol113-elicited SCFA production can help prevent HFD-induced hyperlipidemia and liver steatosis.

\section{Fullerenol NPs show a similar capacity of inulin to promote SCFA-producing bacteria in vitro}

To explore how Fol1 and Fol113 NPs promote SCFAsproducing bacteria survival, we performed a set of investigations in which we analyzed fullerenol NP structure, determined their degradation by gut flora, and carried out in vitro fermentation to assess their direct impacts on gut microbiota. 


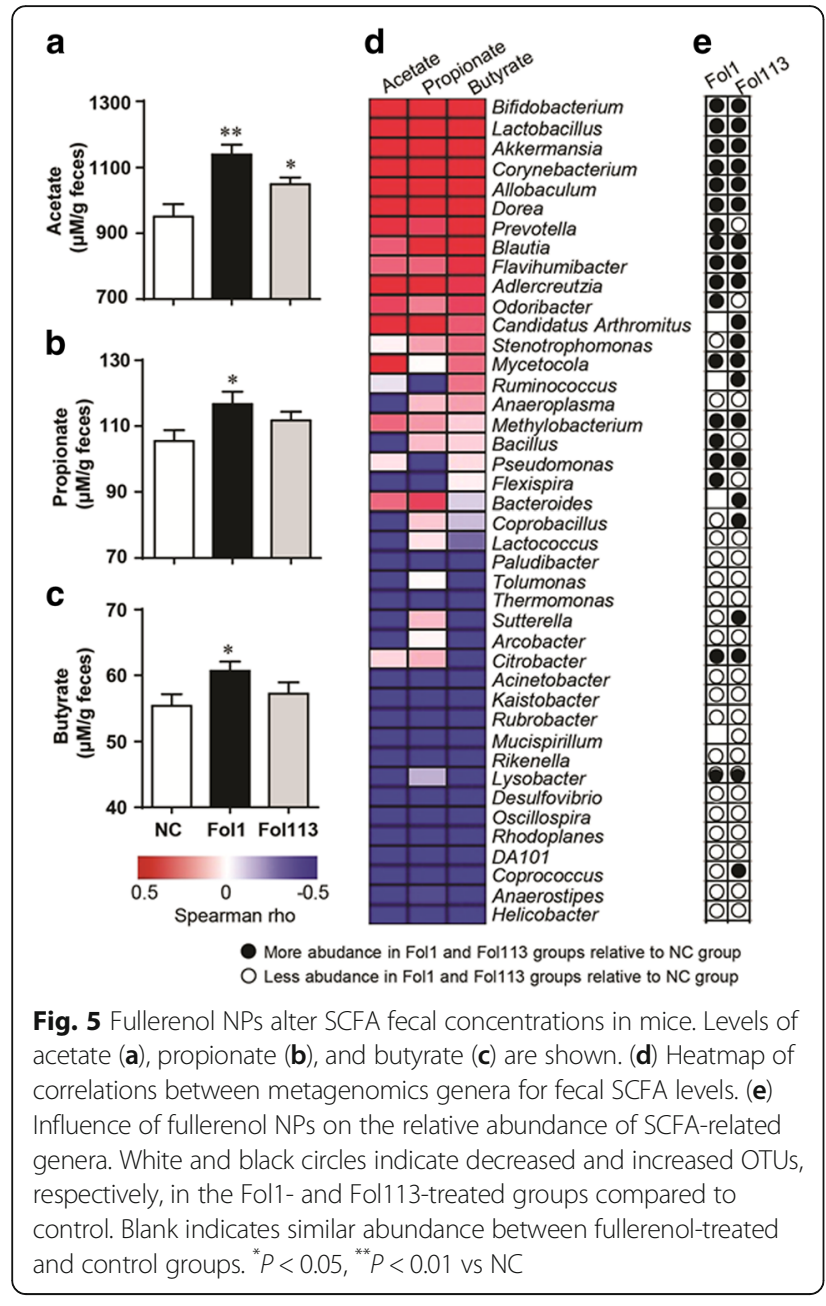

As revealed by synchrotron radiation XPS analysis and FTIR data (Fig. 1 and Additional file 1: Figure S1), fullerenol NPs were rich in complex -OH groups including peroxo groups to hydroxy groups and peroxo groups to epoxy groups [31, 32]. Fol1 and Fol113 can be expressed by the general formulae $\mathrm{C}_{60}(\mathrm{OH})_{7}\left(\mathrm{O}_{8}\right.$ and $\mathrm{C}_{60}(\mathrm{OH})_{11}(\mathrm{O})_{6}$, respectively. There may be furan- and pyran-like structures on fullerenol stereostructures (see Fig. 4b). Since the furan- and pyran-like structures of fullerenol NPs appeared similar to those of the polysaccharides in dietary fiber, which is the main nutrient for SCFAs-producing gut bacteria [33], we hypothesize that Fol1 and Fol113 NPs might function like dietary polysaccharides to promote SCFAs-producing bacteria survival.

Being bacterial nutrients, NPs should be degraded by gut microbes. We therefore incubated the two fullerenol NPs with gut flora and determined whether they could be degraded by gut microbial community. We performed matrix-assisted laser desorption/ionization-time of flight-mass spectrometry (MALDI-TOF-MS) to quantify the fullerenols of $\mathrm{C}_{60}(\mathrm{e} / \mathrm{m}=720)$ with polyhydroxyl $\mathrm{C}_{70}$ $(\mathrm{e} / \mathrm{m}=840)$ as reference (Additional file 1: Figure S5).
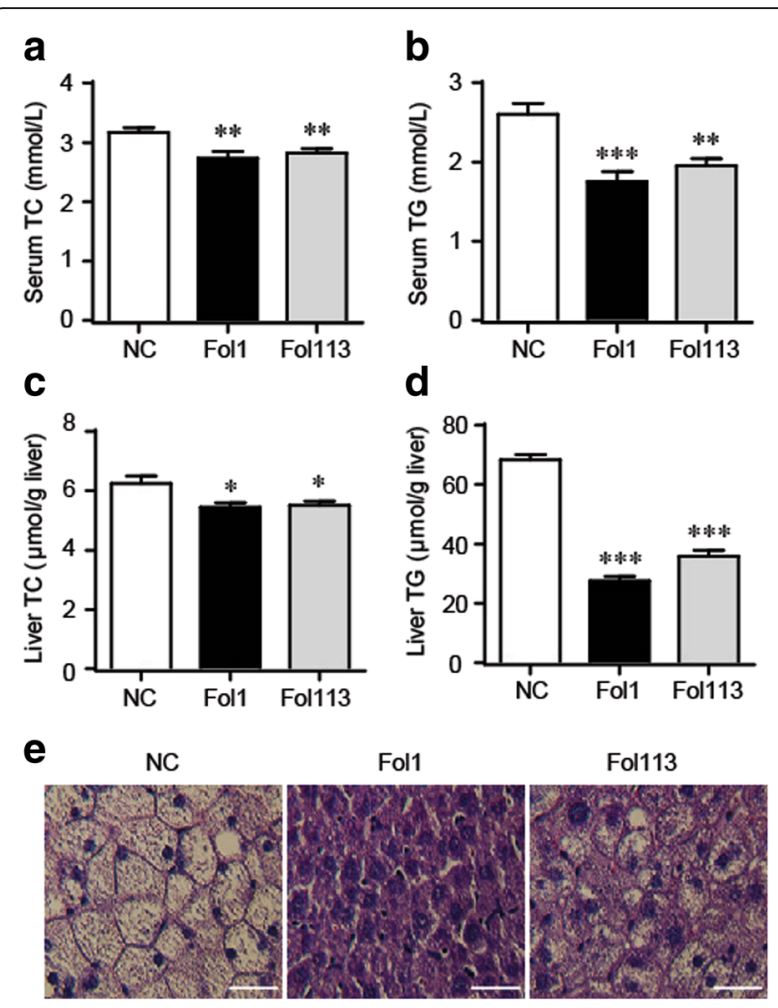

Fig. 6. Fullerenol NPs decrease blood and liver lipids in mice. Blood levels of TC (a) and TG (b), liver levels of TC (c) and TG (d), and representative hematoxylin and eosin staining of the liver (e) are shown. Bar $=50 \mu \mathrm{m} .{ }^{*} P<0.05,{ }^{* *} P<0.01,{ }^{* * *} P<0.001$ vs NC

After fermentation for $48 \mathrm{~h}$, the concentrations of the two fullerenols were reduced by $0.069 \pm 0.0031$ and $0.088 \pm 0.0012 \mathrm{mg} / \mathrm{mL}$, respectively (Fig. 7a). The final concentrations of Fol1 and Fol113 in fermented solution corresponded to $0.037 \pm 0.0031$ and $0.033 \pm 0.0017 \mathrm{mg} /$ $\mathrm{mL}$ (Fig. 7b). Incubating fullerenols with the medium without feces for $48 \mathrm{~h}$ did not alter the measured concentrations. These results imply that Fol1 and Fol113 NPs can be degraded and used by gut microbes.

Inulin is a popular dietary polysaccharide that can promote SCFAs-producing gut microbes. We further performed in vitro fermentation in a micro-anaerobic atmosphere, using inulin as positive control to evaluate the direct effect of fullerenol NPs on SCFA-producing bacteria. Inulin significantly enhanced the relative abundance of SCFA-producing bacteria such as Bifidobacterium and Clostridium cluster IV (Fig. 7c and d), as previously reported [34]. Fermentation with Foll and Fol113 NPs also significantly increased the relative abundance of Clostridium IV and, to a lesser extent, Bifidobacterium (Fig. 7c and d). Another putative SCFA producer, Allobaculum spp., was significantly increased by Fol1 and Fol113 in vitro and in vivo, whereas inulin had minimal effect (Fig. 7e). These results suggest that 

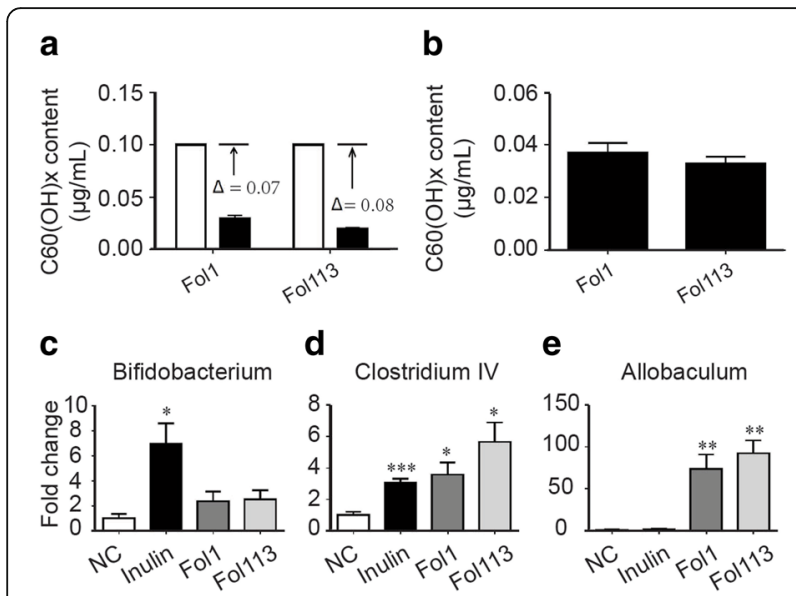

f

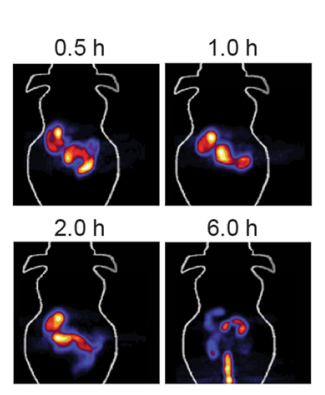

g

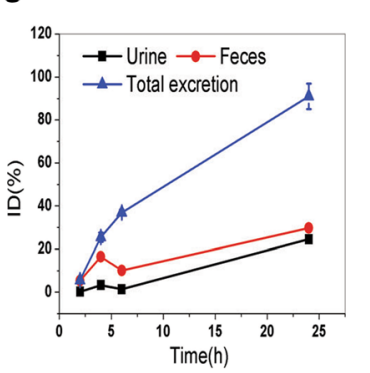

Fig. 7 Fullerenol NPs interact with gut microbiota during in vitro gut flora fermentation. a Fullerenols concentration changes in the fermentation solution; $\mathbf{b}$ effects of the final concentrations of different fullerenols after in vitro fermentation; $\mathbf{c}$-e responses of Bifidobacterium, Clostridium IV, and Allbaculum spp. to inulin and fullerenol NPs; ${ }^{*} P<0.05,{ }^{* *} P<0.01,{ }^{* * *} P<0.001$ vs NC. f PET images of ${ }^{64} \mathrm{Cu}_{-} \mathrm{C}_{60}$ at $0.5,1,2$, and $6 \mathrm{~h}$ after single oral administration. $\mathbf{g}$ Excretion curves of ${ }^{64} \mathrm{Cu}_{-} \mathrm{C}_{60}$ at $1,2,4,6$, and $24 \mathrm{~h}$ after single oral administration. All data points represent three animals per group. The total excretion was the sum of percentages of ${ }^{64} \mathrm{Cu}$ in all the waste $\left(\Sigma_{0-t}\right.$ urine $+\Sigma_{0-t}$ feces $)$ during 0 -t not at the time point

fullerenol NPs possess a similar but not identical capacity to inulin to modulate gut microbiota in vitro.

We also investigated the absorption and excretion of orally administrated fullerenol NPs in mice using positron emission tomography (PET). The microPET images taken $0.5,1,2$, and $6 \mathrm{~h}$ after ${ }^{64} \mathrm{Cu}$-fullerenol administration showed that the signals appeared exclusively in the GI tract (Fig. 7f). The radioactivity level $24 \mathrm{~h}$ after oral administration as not detectable by the PET imaging detector, suggesting that Fol1 and Fol113 NPs distributed mainly in the GI tract and were excreted within $24 \mathrm{~h}$ of oral administration. We further quantified ${ }^{64} \mathrm{Cu}$-fullerenol excretion in feces and urine at $1,2,4,6$, and $24 \mathrm{~h}$ after single oral administration (Fig. 7g). The percentages of ${ }^{64} \mathrm{Cu}$-fullerenols in were calculated by using the equation (Eactivity of excrement/ total activity). The total excretion was the sum of percentages of ${ }^{64} \mathrm{Cu}$ in all the waste $\left(\Sigma_{0-\mathrm{t}}\right.$ urine $+\Sigma_{0-\mathrm{t}}$ feces). Figure $7 \mathrm{~g}$ shows that $\sim 95 \%$ of ${ }^{64} \mathrm{Cu}$-fullerenol was excreted within
$24 \mathrm{~h}$. In other words, few NPs were absorbed by the animals. This is further evidence that fullerenol might indirectly regulate lipid metabolism in the GI tract rather than direct effects in the liver or circulation.

Collectively, this set of in vitro experiments suggests that the effects of fullerenols on lipid metabolism could be attributable to their regulation of gut microbiota. Although the underlying mechanism remains to be elucidated, and the diverse structural effects of Fol1 and Fol113 need further investigation, our results indicate that fullerenol NPs might promote SCFA-producing microbes by functioning as their nutrients. Indeed, clinical studies have shown that SCFAs can substantial decrease TG levels but only achieve a modest decrease in cholesterol [35]. In our study, fullerenol NPs differentially affected TC and TG levels, with greater impacts on TG. Therefore, the increased abundance of SCFAs-producing genera and high fecal SCFA content in fullerenol NPtreated animals verify the microbe-regulating effects of fullerenol NPs in vivo.

\section{Subtly varied surface structures may contribute to the differential effects of Fol 1 and Fol113 NPs on the microbiota} Although Fol1 and Fol113 had similar influences on the overall gut microbiota structure and host metabolism, their impacts on special microbes were discrepant. For instance, diversity analysis by Shannon rarefaction OTU estimates, observed species, Chao1 and Shannon diversity indexes indicated that Fol113 NPs significantly decreased the diversity of the gut microbiota while Fol1 showed no influence (Additional file 1: Figure S6a-S6d). On individual taxon, Fol1 selectively enriched the phyla of Verrucomicrobia, while Fol113 decreased its abundance (Additional file 2: Table S3). The relative percentage of Akkermansia, the major genus in Verrucomicrobia that is known to be beneficial for the prevention and treatment of obesity, dyslipidemia, and diabetes, was increased more than 40-fold in Fol1-treated mice, while there was only a slight increase in Fol113-treated mice (Fig. 2d and Additional file 2: Table S3). While both fullerenol NPs promoted the flourishing of SCFA-producing microbes and increased SCFA production, Fol1 was more effective Fol113 (Figs. 2, 3 and 4). Accordingly, the effect of Fol1 NPs in decreasing TG blood and liver concentrations was greater than that of Fol113 NPs (Fig. 6). We propose that the differential effects of the two fullerenol NPs on special gut microbes and host metabolism might be attributable to subtle variations in their surface structures.

Multiple investigations have demonstrated that NP surface structure profoundly influences their bioactivities $[2,21,36]$. Fullerenols are complicated mixtures of compounds, and their peroxo and hydroxy groups deeply affect their biological activities [3, 37]. Mizuno et al. reported that the in vitro inhibitive effect of fullerenol NPs 
on microbe growth was markedly enhanced with additional surface hydroxyl groups [24, 31, 38]. The Fol1 NPs in the present paper $\left(\mathrm{C}_{60}(\mathrm{OH})_{7}(\mathrm{O})_{8}\right)$ had more peroxo or epoxy groups, while Fol113 NPs $\left(\mathrm{C}_{60}(\mathrm{OH})_{11}(\mathrm{O})_{6}\right)$ had more hydroxy groups. Physiochemical characterization showed that Fol113 NPs were more soluble, more hydrophilic, and formed smaller aggregates in water than Fol1. Therefore, the subtly varied surface structures may contribute to the differential effects of Fol1 and Fol113 NPs on the microbiota.

\section{Conclusions}

Our results provide clear evidence that Fol1 and Fol113 fullerenol NPs can change gut microbiota structure both in vitro and in vivo. They selectively enrich SCFAproducing bacteria and promote SCFA production. The modulating effects of Fol1 and Fol113 NPs on gut microbiota and their metabolites ultimately lead to significant physiological benefits that prevent hyperlipidemia.

\section{Additional files}

Additional file 1: Table S1. The Zeta potential and hydrodynamic sizes of Fol1 and Fol113. Table S2. Oligonucleotide primers used in this work. Figure S1. Physicochemical characterization of fullerenols. (a) Pictures of respective solutions, (b) UV-vis spectra, (c) FTIR spectra, (d) and (e) XPS spectra of Fol 1 and Fol 113. (f) and (g) MALDI-TOF spectra of Fol 1 and Fol 113. Figure S2. The Rarefaction curves (a) and Shannon-Wiener curves (b) indicated a sufficient coverage of the phylotypes by the current sampling number and sequencing depth. Figure S3. OTU Venn analysis. Figure S4. Responses of top 50 abundant OTUs to fullerenols treatment. (a) Heatmap showing the abundance of top 50 OTUs. (b) Represented abacterial taxa information (phylum, family, genus and species) of 50 OTUs from $\mathbf{a}$. White and black circles indicate decreased and increased OTUs, respectively, in the Fol1- and Fol113-treated groups compared to control. Blank indicates similar abundance between fullerenol-treated and control groups. Figure S5. Quantitative analysis of fullerenols in gut flora fermentation solution by MALDI-TOF-MS. (a) MALDI-TOF-MASS spectra of samples; (b) the standard curve of $C_{60} / C_{70}$ and the concentration of $\mathrm{C}_{60}$. Figure $\mathbf{S 6}$. Diversity and richness of the gut microbiota in mice. (a) OTU estimates, (b) Observed species, (c) Chao1 diversity index, (d) Shannon diversity index. Data are represented as means \pm standard error. Differences were assessed by ANOVA and denoted as follows: ${ }^{* * *} P<0.001$. (DOCX $3554 \mathrm{~kb}$ )

Additional file 2: Table S3. Taxon-based summaries for oral administration of fullerenol NPs. (XLSX $152 \mathrm{~kb}$ )

\section{Acknowledgements}

We would like to acknowledge Prof. Kuirexi and other fellow research members at the Beijing Synchrotron Radiation Facility in helping with the XPS work.

\section{Funding}

This work was supported financially by the National Basic Research Program of China (973 Program) (2015CB930104, 2013CB932703), National Natural Science Foundation of China (11405185, 31571028, 31300826, 81402983, 81673663), CAMS Innovation Fund for Medical Sciences (CIFMS) 2016-12M-3-015.

\section{Availability of data and materials}

All data generated or analyzed during this study are included in this published article and its supplementary information files.

\section{Authors' contributions}

JL: Wrote the manuscript, analyzed all data, prepared all tables and figures, and conducted most of the experimental work (e.g., NPs characterizations, 16 s DNA detection, gut microbacteria statistical analysis, pathway analysis). RL: Assisted in conducting animal experiments, the preparation of DNA samples from exposed mice and contributed to the writing of manuscript. $\mathrm{XL}$ : Assisted in the detection of $16 \mathrm{~s}$ DNA and biochemistry analysis. FX: Assisted in conducting animal experiments and the preparation of DNA samples from exposed mice. QZ: Assisted in the $16 \mathrm{~s}$ DNA analysis. YZ: Assisted in the 16 s DNA analysis. SY: Assisted in conducting animal experiments. YC: Assisted in statistical analysis. WG: Conducted physical analyses of NPs. KC: Assisted in statistical analysis. CW: Designed experiments, statistical analysis and contributed to the writing of manuscript. GX: Designed experiments, contributed to the writing of manuscript and supported data interpretation. All authors read and approved the final manuscript.

\section{Ethics approval and consent to participate}

All the animal experiments were performed in accordance with the National Institutes of Health regulations for the care and use of animals in research and were approved by the Medical Ethics Committee of Peking Union Medical College (No. YZS201603004).

\section{Consent for publication}

All authors consent to publication of this manuscript.

\section{Competing interests}

The authors declare that they have no competing interests.

\section{Publisher's Note}

Springer Nature remains neutral with regard to jurisdictional claims in published maps and institutional affiliations.

Received: 12 October 2017 Accepted: 3 January 2018

Published online: 17 January 2018

References

1. Zhang L, Gu FX, Chan JM, Wang AZ, Langer RS, Farokhzad OC. Nanoparticles in medicine: Therapeutic applications and developments. Clin Pharmacol Ther. 2008:835:761-769. <Go to |S|>//WOS:000255288100025.

2. Jiao F, Liu Y, Qu Y, Li W, Zhou GQ, Ge CC, et al. Studies on anti-tumor and antimetastatic activities of fullerenol in a mouse breast cancer model. Carbon. 2010:488:2231-2243. <Go to |SI>://WOS:000277780500012.

3. Meng H, Xing GM, Sun BY, Zhao F, Lei H, Li W, et al. Potent Angiogenesis Inhibition by the Particulate Form of Fullerene Derivatives. Acs Nano. 2010;45:2773-2783. <Go to ISI>://WOS:000277976900037.

4. Roursgaard M, Poulsen SS, Kepley CL, Hammer M, Nielsen GD, Larsen ST. Polyhydroxylated C(60) fullerene (fullerenol) attenuates neutrophilic lung inflammation in mice. Basic Clin Pharmacol Toxicol. 2008;103 4:386-388. $<$ Go to $|S|>$ :/NOS:000259272100015.

5. Han LQ, Wang TQ, Wu JL, Yin XL, Fang H, Zhang N. A facile route to form self-carried redox-responsive vorinostat nanodrug for effective solid tumor therapy. Int J Nanomedicine. 2016;11:6003-6022. <Go to |SI>://WOS: 000387443100001

6. Osuka S, Van Meir EG. CANCER THERAPY Neutrophils traffic in cancer nanodrugs. Nat Nanotechnology. 2017;12 7:616-618. <Go to ISI>://WOS: 000404874000009

7. Clemente JC, Ursell LK, Parfrey LW, Knight R. The Impact of the Gut Microbiota on Human Health: An Integrative View. Cell. 2012;148 6:12581270. $<$ Go to $|S|>: / / W O S: 000301889500020$.

8. Qin JJ, Li YR, Cai ZM, Li SH, Zhu JF, Zhang F, et al. A metagenome-wide association study of gut microbiota in type 2 diabetes. Nature. 2012;490 7418:55-60. <Go to ISI >://WOS:000309446800031.

9. Ridaura VK, Faith JJ, Rey FE, Cheng JY, Duncan AE, Kau AL, et al. Gut Microbiota from Twins Discordant for Obesity Modulate Metabolism in Mice. Science. 2013;341 6150:1079-1U49. <Go to ISI>://WOS: 000323933100034

10. Shin NR, Lee JC, Lee HY, Kim MS, Whon TW, Lee MS, et al. An increase in the Akkermansia spp. population induced by metformin treatment improves glucose homeostasis in diet-induced obese mice. Gut. 2014;63(5): 
727-35. https://doi.org/10.1136/gutjnl-2012-303839. https://www.ncbi.nlm. nih.gov/pmc/articles/PMC4585776/

11. Zhang $X$, Zhao YF, Xu J, Xue ZS, Zhang MH, Pang XY, et al. Modulation of gut microbiota by berberine and metformin during the treatment of highfat diet-induced obesity in rats. Scientific Reports. 2015;5:14405. <Go to $|S|>: / /$ WOS:000361598400001.

12. Chang CJ, Lin CS, Lu CC, Martel J, Ko YF, Ojcius DM, et al. Ganoderma lucidum reduces obesity in mice by modulating the composition of the gut microbiota. Nat Commun. 2015;6:7489. <Go to |SI>:/WOS:000357178100009.

13. Zhang DY, Ji HF, Liu H, Wang SX, Wang J, Wang YM. Changes in the diversity and composition of gut microbiota of weaned piglets after oral administration of Lactobacillus or an antibiotic. Appl Microbiol Biotechnol. 2016;100 23:10081-10093. <Go to |SI>://WOS:000387656500023.

14. Dao MC, Everard A, Aron-Wisnewsky J, Sokolovska N, Prifti E, Verger EO, et al. Akkermansia muciniphila and improved metabolic health during a dietary intervention in obesity: relationship with gut microbiome richness and ecology. Gut. 2016;65 3:426-436. <Go to |S|>:/WOS:000371321700011.

15. van den Brule S, Ambroise J, Lecloux H, Levard C, Soulas R, De Temmerman $\mathrm{PJ}$, et al. Dietary silver nanoparticles can disturb the gut microbiota in mice. Part Fibre Toxicol. 2016;13:38-54. <Go to ISI>://WOS:000379264300001.

16. Christophersen DV, Jacobsen NR, Andersen MH, Connell SP, Barfod KK, Thomsen MB, et al. Cardiovascular health effects of oral and pulmonary exposure to multi-walled carbon nanotubes in ApoE-deficient mice. Toxicology. 2016;371:29-40. https://doi.org/10.1016/j.tox.2016.10.003. http:// www.sciencedirect.com/science/article/pii/S0269749113003187

17. Zhang L, Petersen EJ, Habteselassie MY, Mao L, Huang Q. Degradation of multiwall carbon nanotubes by bacteria. Environ Pollut. 2013;181:335-9. https://doi.org/10.1016/j.envpol.2013.05.058. https://link.springer.com/article/ 10.1007/s11356-016-6474-y

18. Yausheva capital le C, Sizova capital le C, Lebedev S, Skalny A, Miroshnikov S, Plotnikov A, et al. Influence of zinc nanoparticles on survival of worms Eisenia fetida and taxonomic diversity of the gut microflora. Environ Sci Pollut Res Int. 2016;23(13):13245-54. https://doi.org/10.1007/s11356-0166474-y. http://www.tandfonline.com/doi/abs/10.3109/17435390.2015. 1078854

19. Wilding LA, Bassis CM, Walacavage $K$, Hashway S, Leroueil PR, Morishita M, et al. Repeated dose (28-day) administration of silver nanoparticles of varied size and coating does not significantly alter the indigenous murine gut microbiome. Nanotoxicology. 2016;10(5):513-20. https://doi.org/10.3109/ 17435390.2015.1078854. https://particleandfibretoxicology.biomedcentral. com/articles/10.1186/s12989-016-0149-1

20. van den Brule S, Ambroise J, Lecloux H, Levard C, Soulas R, De Temmerman PJ, et al. Dietary silver nanoparticles can disturb the gut microbiota in mice. Part Fibre Toxicol. 2016;13(1):38. https://doi.org/10.1186/s12989-016-0149-1. http://pubs.acs.org/doi/abs/10.1021/nn900318y

21. Chaudhuri P, Paraskar A, Soni S, Mashelkar RA, Sengupta S. FullerenolCytotoxic Conjugates for Cancer Chemotherapy. Acs Nano. 2009;3 9:25052514. <Go to ISI>://WOS:000269988600011.

22. Aoshima H, Kokubo K, Shirakawa S, Ito M, Yamana S, Oshima T. Antimicrobial Activity of Fullerenes and Their Hydroxylated Derivatives. Biocontrol Sci. 2009;14 2:69-72. <Go to |SI>://WOS:000267195400005.

23. Yin $R$, Wang M, Huang $Y Y$, Landi G, Vecchio D, Chiang LY, et al. Antimicrobial photodynamic inactivation with decacationic functionalized fullerenes: oxygen-independent photokilling in presence of azide and new mechanistic insights. Free Radic Biol Med. 2015;79:14-27. https:/doi.org/10.1016/j. freeradbiomed.2014.10.514. http://www.sciencedirect.com/science/article/pii/ S0008622311007342

24. Li J, Zhang MY, Sun BY, Xing GM, Song Y, Guo HL, et al. Separation and purification of fullerenols for improved biocompatibility. Carbon. 2012;50 2: 460-469. <Go to ISI>://WOS:000297397700014.

25. Mo H. China PsRo. Chinese National Formulary. 2005:535.http://iopscience. iop.org/article/10.1088/0957-4484/27/15/155101/meta

26. Li J, Yang WJ, Cui RL, Wang DL, Chang YA, Gu WH, et al. Metabolizer in vivo of fullerenes and metallofullerenes by positron emission tomography. Nanotechnology. 2016;27 15:1-12. <Go to ISI>://WOS:000371343500004.

27. Perez-Lopez E, Cela D, Costabile A, Mateos-Aparicio I, Ruperez P. In vitro fermentability and prebiotic potential of soyabean Okara by human faecal microbiota. Br J Nutr. 2016;116 6:1116-1124. <Go to |S|>://WOS: 000382996100017
28. Hamady M, Lozupone C, Knight R. Fast UniFrac: facilitating high-throughput phylogenetic analyses of microbial communities including analysis of pyrosequencing and PhyloChip data. Isme J. 2010:4 1:17-27. <Go to ISI>//MOS:000273350200002.

29. Arumugam M, Raes J, Pelletier E, Le Paslier D, Yamada T, Mende DR, et al. Enterotypes of the human gut microbiome. Nature. 2011;473(7346):174-80. https:/ doi.org/10.1038/nature09944. http://science.sciencemag.org/content/341/6145/569

30. Smith PM, Howitt MR, Panikov N, Michaud M, Gallini CA, Bohlooly-Y M, et al. The Microbial Metabolites, Short-Chain Fatty Acids, Regulate Colonic T-reg Cell Homeostasis. Science. 2013;341 6145:569-573. <Go to ISI>://WOS: 000322586700059

31. Wang ZZ, Lu ZH, Zhao YL, Gao XF. Oxidation-induced water-solubilization and chemical functionalization of fullerenes C-60, Gd@C-60 and Gd@C-82: atomistic insights into the formation mechanisms and structures of fullerenols synthesized by different methods. Nanoscale. 2015;7 7:29142925. $<$ Go to $|S|>: / /$ WOS:000349473200013.

32. Yin JJ, Lao F, Fu PP, Wamer WG, Zhao YL, Wang PC, et al. The scavenging of reactive oxygen species and the potential for cell protection by functionalized fullerene materials. Biomaterials. 2009;30 4:611-621. <Go to $|S|>/ /$ WOS:000262065500023.

33. Kim M, Qie YQ, Park J, Kim CH. Gut Microbial Metabolites Fuel Host Antibody Responses. Cell Host Microbe. 2016;20 2:202-214. <Go to ISI>:// WOS:000381587500012.

34. Rau M, Rehman A, Levels H, Weiss J, Beyersdorf N, Rosenstiel P, et al. Shortchain fatty acids and SCFA-producing bacteria in NAFLD patients are associated with an increased Th17/rTreg ratio and hepatic disease progression. Journal of Hepatology. 2017;66 1:S600-S60S. <Go to ISI>://WOS: 000401056601474

35. Sharma S, Puri S. Prebiotics and Lipid Metabolism: A Review. Altern Ther Health Med. 2015;21:34-42. <Go to ISI>://WOS:000368749200005.

36. Wang ZZ, Wang SK, Lu ZH, Gao XF. Syntheses, Structures and Antioxidant Activities of Fullerenols: Knowledge Learned at the Atomistic Level. Journal of Cluster Science. 2015;26 2:375-388. <Go to ISI>://WOS:000351310300006.

37. Zhang $G$, Liu Y, Liang $D H$, Gan LB, Li Y. Facile Synthesis of Isomerically Pure Fullerenols and Formation of Spherical Aggregates from $\mathrm{C}-60(\mathrm{OH})(8)$. Angew Chem-Int Ed. 2010;49 31:5293-5295. <Go to |SI>:/NOS: 000280464200010

38. Topping DL, Clifton PM. Short-chain fatty acids and human colonic function: Roles of resistant starch and nonstarch polysaccharides. Physiol Rev. 2001;81 3:1031-1064. <Go to |S|>://WOS:000169570800003.

\section{Submit your next manuscript to BioMed Central and we will help you at every step:}

- We accept pre-submission inquiries

- Our selector tool helps you to find the most relevant journal

- We provide round the clock customer support

- Convenient online submission

- Thorough peer review

- Inclusion in PubMed and all major indexing services

- Maximum visibility for your research

Submit your manuscript at www.biomedcentral.com/submit
) Biomed Central 